\title{
PREVALÊNCIA DE SOBREPESO E OBESIDADE EM ESCOLARES COM IDADE DE 7 A 17 ANOS, RESIDENTES NOS MUNICÍPIOS PERTENCENTES À SECRETARIA DE DESENVOLVIMENTO REGIONAL DE SÃO MIGUEL DO OESTE/SC
}

Andréa Jaqueline Prates Ribeiro, Universidade do Oeste de Santa Catarina - UNOESC, São Miguel, Santa Catarina - Brasil

Sandra Fachineto, Universidade do Oeste de Santa Catarina - UNOESC, São Miguel, Santa Catarina - Brasil

Sandro Claro Pedrozo, Universidade do Oeste de Santa Catarina-UNOESC, São Miguel, Santa Catarina - Brasil

Ana Paula Trentin, Universidade do Oeste de Santa Catarina - UNOESC, São Miguel, Santa Catarina - Brasil

Cassiane Zapoani, Universidade do Oeste de Santa Catarina - UNOESC, São Miguel, Santa Catarina - Brasil

\section{RESUMO}

O objetivo deste estudo foi verificar prevalência de sobrepeso e obesidade em escolares de 7 a 17 anos, residentes nos municípios pertencentes à Secretaria de Desenvolvimento Regional (SDR) de São Miguel do Oeste/SC. Participaram do presente estudo 1.728 escolares, sendo 902 do gênero masculino e 826 do feminino. O IMC foi calculado e classificado conforme o procedimento utilizado pelo PROESP-BR. A análise de frequiência foi utilizada para verificar a distribuição de sobrepeso e obesidade e o teste do quiquadrado $\left(\mathrm{x}^{2}\right)$ para atestar as diferenças entre os valores percentuais (\%) no que diz respeito ao gênero, município e IMC. O nível de significância adotado foi $\mathrm{P} \leq 0,05$. Dos 1.728 escolares, constatou-se sobrepeso em 351 (20,3\%) e obesidade em 91 (5,3\%). Ao comparar os gêneros, verificou-se que a prevalência de sobrepeso foi maior no gênero masculino do que no feminino, principalmente nos municípios de Belmonte/SC $(\mathrm{P}=0,021)$, Descanso/SC $(\mathrm{P}=0,010)$ e São Miguel do Oeste/SC $(\mathrm{P}=0,009)$. Em relação à obesidade, não foram observadas diferenças significativas entre os sexos. Dessa forma, conclui-se que em ambos os gêneros, a prevalência de sobrepeso e obesidade em escolares de 7 a 17 anos, residentes nos municípios pertencentes a SDR de São Miguel do Oeste/SC, é considerada alta, pois atinge uma fração de $24,8 \%$ da população feminina e $26,4 \%$ da masculina.

Palavras-Chave: Sobrepeso; Obesidade; Escolares.

\section{PREVALENCE OF OVERWEIGHT AND OBESITY IN SCHOOLCHILDREN AGED 7 TO 17 YEARS, RESIDENTS IN THE CITIES BELONGING TO THE DEVELOPMENT SECRETARIAT REGIONAL OF SÃO MIGUEL DO OESTE/SC}




\begin{abstract}
The objective of this study was to determine the prevalence of overweight and obesity in schoolchildren aged 7 to 17 years, residents in the municipalities belonging to the Regional Development Secretariat (RDS) of São Miguel do Oeste/SC. The study included 1728 school children, 902 males and 826 females. BMI was calculated and classified according to the procedure used by PROESP-BR. Frequency analysis was used to investigate the distribution of overweight and obesity and the chi-square (x2) to demonstrate the differences between the percentages $(\%)$ with regard to gender, county and BMI. The level of significance was $\mathrm{P} \leq 0.05$. Of the 1,728 students, was found in 351 overweight $(20.3 \%)$ and obesity in $91(5.3 \%)$. When comparing genders, it was found that the prevalence of overweight was higher in males than in females, mainly in the municipalities of Belmonte/SC $(\mathrm{P}=0.021)$, Descanso/SC $(\mathrm{P}=0.010)$ and São Miguel do Oeste/SC $(\mathrm{P}=$ 0.009). In relation to obesity, there were no significant differences between the sexes. Thus, we conclude that in both genders, the prevalence of overweight and obesity in schoolchildren aged 7 to 17 years, residents in the municipalities belonging to the RDS of São Miguel do Oeste/SC, is considered high, because reaches a fraction of $24,8 \%$ of female population and $26,4 \%$ of male population.
\end{abstract}

Key-Words: Overweight; Obesity; Students. 


\section{INTRODUÇÃO}

A obesidade é definida como a quantidade excessiva de gordura corporal apresentada por um indivíduo. ${ }^{1}$ Alguns autores ${ }^{2}$ referem-se à obesidade como uma condição que segue um agrupamento de doenças crônicas não transmissíveis e está associada à presença de comorbidades como a resistência a insulina, intolerância a glicose, dislipidemia, hipertensão, diabetes tipo II e câncer.

Quanto à obesidade na infância e adolescência, a mesma pode ocorrer por inúmeras causas e traz implicações na saúde geral, no funcionamento da família, nas emoções entre outros aspectos. O profissional que lida com essa população deve levar em consideração todos esses aspectos envolvidos no processo da obesidade. ${ }^{3}$

O sobrepeso e a obesidade na infância são considerados como epidemia, tanto nos países desenvolvidos quanto naqueles que estão em desenvolvimento e atingem meninos e meninas, indistintamente, o que se associa a problemas de saúde mais freqüentes e a custos cada vez mais elevados. ${ }^{4}$ A ocorrência da obesidade na infância e adolescência favorece sua persistência na vida adulta. ${ }^{5}$

O sedentarismo e a alimentação inadequada são alguns dos possíveis fatores responsáveis pelo aumento da incidência da obesidade na infância. A permanência da obesidade na vida adulta tem como conseqüência, altos riscos de desenvolver doenças cardiovasculares e diabetes, além de acarretar em distúrbios psicológicos, isolamento, depressão e baixa autoestima. Portanto, que a prevenção da obesidade deve ter como foco, principalmente, orientações à prática de atividades físicas regulares e a adoção de uma alimentação saudável. ${ }^{6}$

A grande preocupação mundial é o impacto econômico global que esses futuros adultos obesos poderão causar. Assim sendo, definir em que condições e quais populações estão mais propicias ao desenvolvimento da obesidade, é importante para que o seu controle e combate possam ser direcionados de uma forma eficaz. ${ }^{7}$ 
Para tanto, o objetivo deste estudo foi o de verificar e comparar a prevalência de sobrepeso e obesidade em escolares com idade de 7 a 17 anos, de ambos os sexos, residentes nos municípios que fazem parte da SDR de São Miguel do Oeste/SC.

\section{METODOLOGIA}

Esta pesquisa caracterizou-se como descritiva e a amostra foi composta por 1.728 escolares, sendo 902 do sexo masculino e 826 do sexo feminino, residentes na SDR de São Miguel do Oeste/SC, conforme mostra o Tabela 1.

Tabela 1- Distribuição da amostra por sexo e por municípios da SDR de São Miguel do

Oeste/SC

\begin{tabular}{lllll}
\hline & \multicolumn{2}{l}{ Sexo Feminino } & \multicolumn{2}{l}{ Sexo Masculino } \\
\cline { 2 - 5 } & $\boldsymbol{n}$ & \% (IC 95\%) & $\boldsymbol{n}$ & \% (IC 95\%) \\
\cline { 2 - 5 } Bandeirante & 30 & $36,1(25,8-46,4)$ & 53 & $63,9(53,6-74,2)$ \\
Belmonte & 60 & $62,5(52,8-72,2)$ & 36 & $37,5(27,8-47,2)$ \\
Barra Bonita & 82 & $51,3(43,6-59,0)$ & 78 & $48,7(41,0-56,4)$ \\
Descanso & 91 & $42,9(36,2-49,6)$ & 121 & $57,1(50,4-63,8)$ \\
Guaraciaba & 138 & $51,3(45,3-57,3)$ & 131 & $48,7(42,7-54,7)$ \\
São Miguel do Oeste & 425 & $46,6(43,4-49,8)$ & 483 & $53,2(50,0-56,4)$ \\
Total & 826 & $47,8(44,4-51,2)$ & 902 & $52,2(48,9-55,5)$ \\
\hline
\end{tabular}

Primeiramente, fez-se contato com a Secretaria de Educação de cada Município informando a natureza e propósito do trabalho. Após autorização foram encaminhados para os diretores das escolas indicadas pela Secretaria de Educação, o Termo de Consentimento Livre e Esclarecido (TCLE) para que os pais ou responsáveis assinassem, autorizando a participação dos escolares.

Em seguida, os alunos foram submetidos a medidas de peso e estatura, realizadas na própria dependência da escola em uma sala reservada. Com posse das medidas, o índice de massa corporal (IMC), que representa o peso dividido pela altura ao quadrado $\left(\mathrm{kg} / \mathrm{m}^{2}\right)$, foi calculado e classificado conforme o procedimento utilizado pelo Projeto Esporte Brasil, PROESP-BR, ${ }^{8}$ que utiliza como referência os valores críticos para a classificação do estado nutricional para crianças e jovens brasileiros propostos por Conde e Monteiro. ${ }^{9}$ De 
acordo com esses critérios, as crianças e jovens brasileiros são classificados em 4 categorias, conforme o IMC: "Baixo Peso", "Normal”, "Sobrepeso" e "Obesidade”.

Para análise destes dados foi utilizado o programa estatístico computacional SPSS, versão 13.0. Os procedimentos estatísticos corresponderam à análise de frequiência para avaliar a distribuição da prevalência de sobrepeso e obesidade entre os municípios da SDR de São Miguel do Oeste, SC e entre os sexos. O teste qui-quadrado $\left(X^{2}\right)$ foi utilizado para atestar diferenças entre os valores percentuais (\%) observados nos respectivos intervalos de confiança (IC 95\%) quanto ao sexo, classificação de IMC e municípios da SDR de São Miguel do Oeste/SC. O nível de significância adotado para as inferências estatísticas foi $\mathrm{P} \leq 0,05$.

\section{RESULTADOS}

Dos 1.728 escolares da rede pública de ensino da SDR de São Miguel do Oeste, SC, de ambos os sexos, com idade entre 7 e 17 anos, analisados e classificados por meio do IMC (GAYA; SILVA, 2007), foram observadas as prevalências de Sobrepeso em 351 escolares (20,3\%/ IC 95\%: 18,4\% - 22,2\%) e de Obesidade em 91 escolares (5,3\%/ IC 95\%: 4,2\% 6,4\%), como mostra a Tabela 2. A maior parte da amostra, correspondente a 1.270 escolares (73,5\%/ IC 95\%: 71,4\% - 75,6\%), foi classificada como Normal e o restante, 16 escolares (0,9\%/ IC 95\%: 0,5\% - 1,3\%), como sendo de Baixo Peso.

Em relação às classificações de IMC (Baixo Peso, Normal, Sobrepeso e Obesidade) utilizadas no presente estudo, pode-se observar (TABELA 2) que somente a prevalência de sobrepeso apresentou diferença significativa $\left(X^{2}{ }_{S P * S D R}: \mathrm{P}=0,008\right)$ no que se refere a comparação entre os municípios da SDR São Miguel do Oeste, SC. 
Tabela 2 - Distribuição dos escolares da SDR São Miguel do Oeste, SC conforme classificação de $\mathrm{IMC}^{8}$ : baixo peso, normal, sobrepeso e obesidade

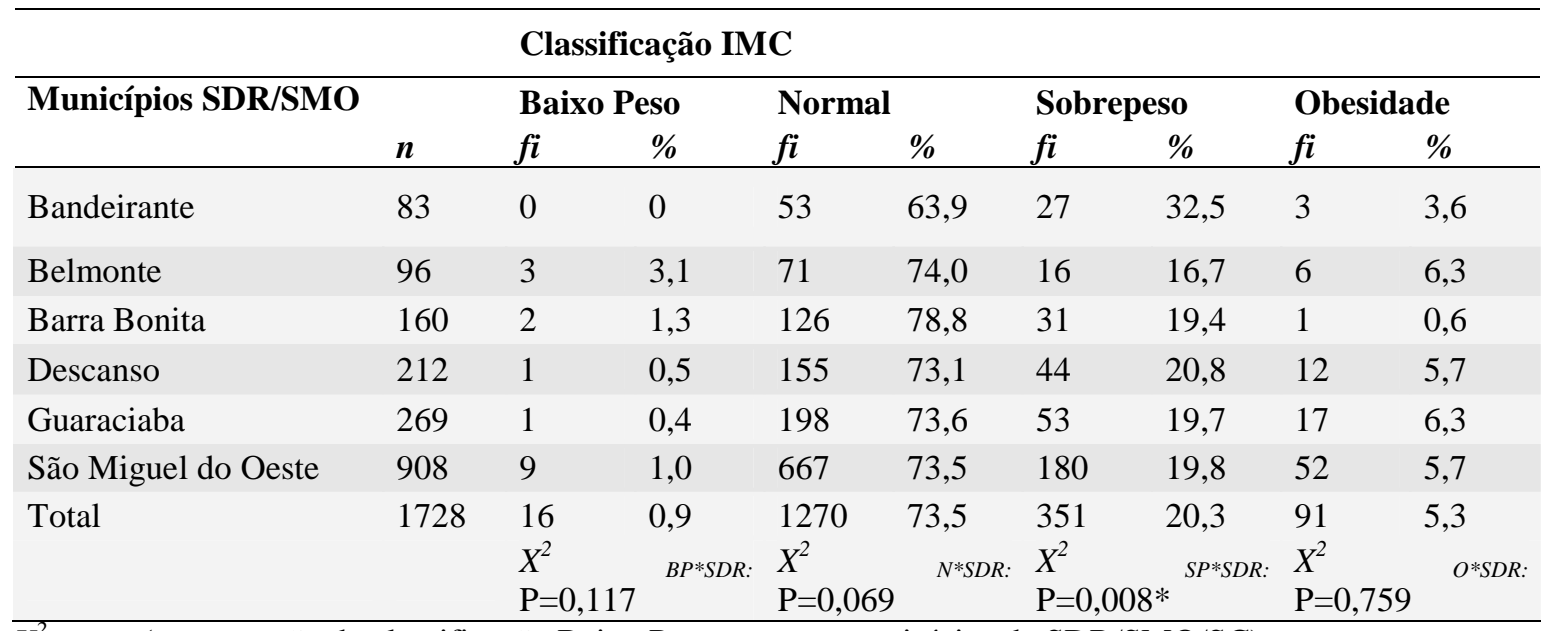

$X^{2}{ }_{B P * S D R}$ (comparação da classificação Baixo Peso entre os municípios da SDR/SMO/SC).

$X^{2}{ }_{N * S D R}$ (comparação da classificação Normal entre os municípios da SDR/SMO/SC).

$X_{S P * S R}^{2}$ (comparação da classificação Sobrepeso entre os municípios da SDR/SMO/SC).

$X^{2}{ }_{O * S D R}$ (comparação da classificação Obesidade entre os municípios da SDR/SMO/SC).

O Gráfico 1 apresenta a freqüência relativa (\%) da prevalência de Sobrepeso e Obesidade, comparada entre os municípios da SDR de São Miguel do Oeste/SC. Pode-se observar que a prevalência de Sobrepeso no município de Bandeirante $(32,5 \%)$ se sobressai em relação aos outros municípios, ou seja, neste município, 27 dos 83 indivíduos se encontram de acordo com esta classificação. O que também chama atenção, no Gráfico 1, é o baixo percentual de prevalência de Obesidade encontrado no município de Barra Bonita $(0,6 \%)$. Neste município, somente 1 dos 160 indivíduos com idade de 7 a 17 anos, de ambos os sexos, apresentou-se obeso.

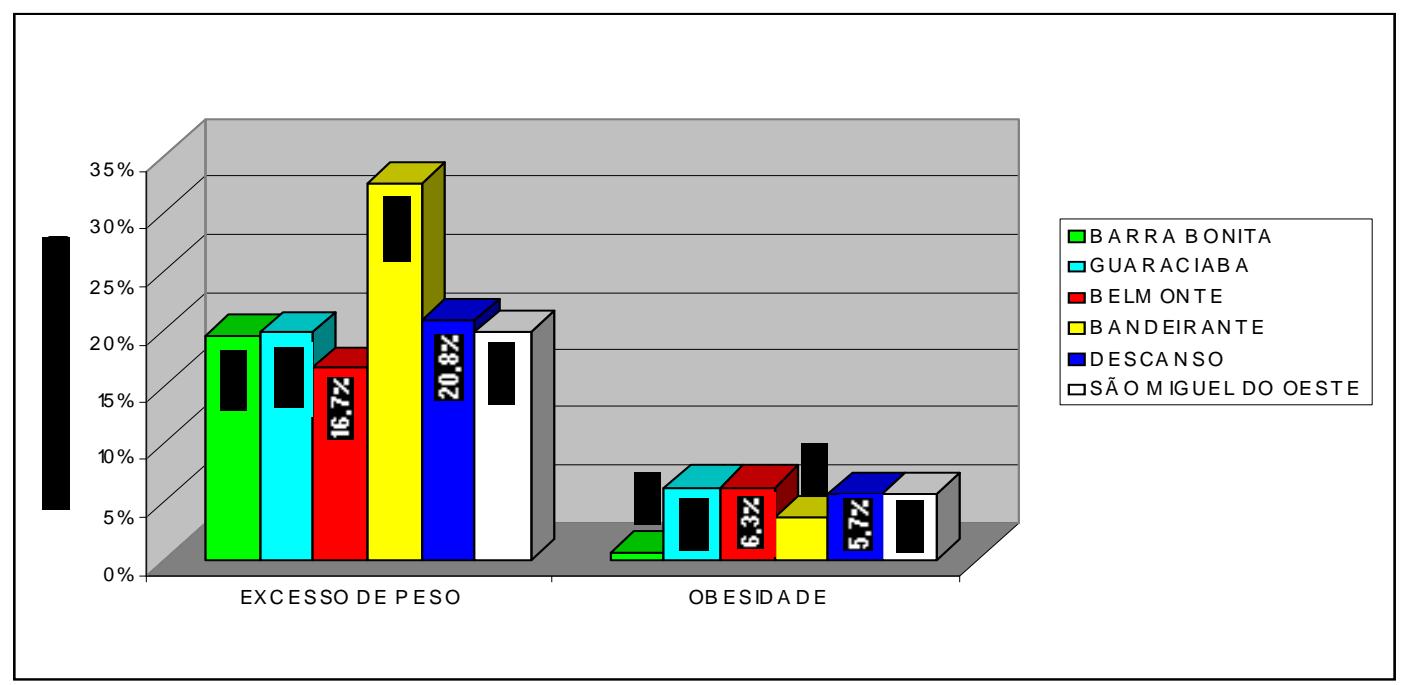

Gráfico 1: Prevalência de Sobrepeso e Obesidade nos municípios da SDR de São Miguel do Oeste, SC. 
Ao analisar a amostra separadamente por sexo (TABELA 3), pode-se observar que a prevalência de Sobrepeso para o sexo masculino $(22,2 \%)$ se apresentou mais elevada $(\mathrm{P} \leq 0,05)$ do que para o feminino $(18,3 \%)$. Entretanto, o mesmo não foi observado no que se refere à prevalência de Obesidade, pois não foram constatadas diferenças significativas $(\mathrm{P} \leq 0,05)$ ao comparar o sexo feminino $(6,5 \%)$ e o sexo masculino $(4,1 \%)$.

Tabela 3 - Prevalência de sobrepeso e obesidade entre os escolares matriculados na rede de ensino pública da SDR de São Miguel do Oeste, SC em ambos os sexos

\begin{tabular}{|c|c|c|c|c|c|c|}
\hline & \multicolumn{3}{|l|}{ Sobrepeso } & \multicolumn{3}{|l|}{ Obesidade } \\
\hline & Sexo & Sexo & & Sexo & Sexo & \\
\hline & Feminino & Masculino & & Feminino & Masculino & \\
\hline & $f(\%)$ & $f(\%)$ & $P$ & $f(\%)$ & $f(\%)$ & $P$ \\
\hline Bandeirante & $11(36,7 \%)$ & $16(30,2 \%)$ & 0,442 & $2(6,7 \%)$ & $1(1,9 \%)$ & 1,000 \\
\hline Belmonte & $13(21,7 \%)$ & $3(8,3 \%)$ & $0,021 *$ & $2(3,3 \%)$ & $4(11,1 \%)$ & 0,688 \\
\hline Barra Bonita & $17(20,7 \%)$ & $14(17,9 \%)$ & 0,720 & $1(1,2 \%)$ & $0(0,0 \%)$ & 1,000 \\
\hline Descanso & $13(14,3 \%)$ & $31(25,6 \%)$ & $0,010 *$ & $7(7,7 \%)$ & $5(4,1 \%)$ & 0,774 \\
\hline Guaraciaba & $25(18,1 \%)$ & $28(21,4 \%)$ & 0,784 & $11(8,0 \%)$ & $6(4,6 \%)$ & 0,332 \\
\hline $\begin{array}{l}\text { São Miguel } \\
\text { Oeste }\end{array}$ & ${ }_{72}^{\text {do }}(16,9 \%)$ & $108(22,4 \%)$ & $0,009 *$ & $31(7,3 \%)$ & $21(4,3 \%)$ & 0,212 \\
\hline Total & $151(18,3 \%)$ & $200(22,2 \%)$ & $0,010^{*}$ & $5,4(6,5 \%)$ & $37(4,1 \%)$ & 0,093 \\
\hline
\end{tabular}

$* \mathrm{P} \leq 0,05$.

Pode-se observar na Tabela 3, que ao comparar os sexos por municípios, a prevalência de Sobrepeso nos municípios de Belmonte $(\mathrm{P}=0,021)$, Descanso $(\mathrm{P}=0,010)$ e São Miguel do Oeste $(\mathrm{P}=0,009)$, para o sexo masculino, apresentou-se mais elevada $(\mathrm{P} \leq 0,05)$. Em relação à prevalência de obesidade, não foram observadas diferenças significativas entre o sexo masculino e o sexo feminino.

\section{DISCUSSÃO}

Ao confrontar os resultados do presente estudo com os de outros estudos nacionais, no que se refere à prevalência de Sobrepeso e Obesidade em escolares, encontramos uma pesquisa realizada por Soar et al., ${ }^{10}$ com uma amostra de 419 escolares com faixa etária entre 7 e 9 
anos de idade, de uma escola pública de Florianópolis. Neste estudo, verificou-se maior prevalência de sobrepeso e obesidade no sexo masculino, embora não apresentando diferença estatisticamente significativa entre os sexos, o que aponta concordância com a prevalência de sobrepeso em alunos do sexo masculino do presente estudo. Quanto aos índices de obesidade, também não houve diferença estatisticamente significativa no presente estudo, no entanto, a prevalência de obesidade foi maior no sexo feminino, enquanto que o grupo escolar de Florianópolis apresentou maior prevalência de obesidade no sexo masculino. Isso possivelmente se deve ao fato de os critérios para classificação para o IMC não terem sido os mesmos, sendo que no estudo de Soar et al. ${ }^{10}$ foram utilizadas as recomendações de Cole et al. ${ }^{11}$ no diagnóstico de sobrepeso e obesidade infantil, enquanto que em nosso estudo foi utilizada a tabela proposta por Gaya e Silva. ${ }^{8}$

Quanto à prevalência de Obesidade, em comparação com estudo realizado por Souza Leão et al., ${ }^{12}$ com 255 alunos entre 5 e 10 anos de escolas públicas de Salvador, Bahia, observou-se que nas escolas públicas a prevalência de obesidade foi maior no sexo masculino entre os alunos de 9 e 10, contrariamente do nosso estudo que não apresentou diferenças significativas entre os sexos. Os autores afirmam que esta faixa etária, entre 9 e 10 anos, é um período de intenso anabolismo e por isso torna propício o desenvolvimento da obesidade. Vale ressaltar que esta discrepância nos resultados de ambos os estudos pode, talvez, ser atribuída ao fato de as amostras de escolares pertencerem a regiões distintas do país: região Nordeste, e, no caso do presente estudo, região Sul. Deve-se considerar, então, inúmeros fatores que podem vir a influenciar os resultados, tais como regionais, climáticos, culturais, etc. Outro fator relevante a ser destacado diz respeito aos diferentes critérios de classificação para o IMC adotados nos estudos, sendo que Souza Leão et al. ${ }^{12}$ seguiram as recomendações de Himes e Dietz, ${ }^{13}$ enquanto que no presente estudo, adotou-se a tabela de classificação de Gaya e Silva. ${ }^{8}$

Em outro estudo, realizado por Neutzling et al., ${ }^{14}$ com 13.715 adolescentes brasileiros com idade entre 10 e 19 anos, a prevalência maior de obesidade se apresentou no grupo de adolescentes do sexo feminino. Novamente houve discrepância em relação aos resultados do presente estudo, pois não ocorreram diferenças significativas entre o sexo feminino e masculino quanto à prevalência de obesidade. Esta diferença entre os resultados de ambos os estudos pode ser atribuída ao fato de a amostra do estudo de Neutzling et al. ${ }^{14}$ ser restrita 
a escolares entre 10 e 19 anos, enquanto que a do presente estudo abrange a faixa etária de 7 a 17 anos.

À nível nacional, há alguns estudos publicados nas áreas da saúde, nutrição e pediatria, abordando a prevalência de sobrepeso e obesidade em adolescentes escolares. Os resultados encontrados nestes estudos mostram que no estado do Rio de Janeiro, na cidade de Niterói, Fonseca, Sichieri e Veiga ${ }^{15}$ avaliaram a prevalência de obesidade em 391 alunos de classe média, a qual foi observada com maior frequiência no sexo feminino, se igualando aos resultados do presente estudo, onde embora sem diferenças estatisticamente significativas, o sexo feminino apresentou valores percentuais maiores que os do sexo masculino, quanto a prevalência de obesidade.

Em Recife, Balaban e Silva ${ }^{16}$ realizaram estudo com 430 estudantes de 10 a 19 anos de uma escola de rede privada, onde verificaram que o sobrepeso, bem como a obesidade, apresentou maior prevalência nos escolares do sexo masculino. Quanto ao sobrepeso, os resultaram se igualaram ao nosso estudo, mas se diferiu em relação à prevalência de obesidade, pois não encontramos diferenças estatisticamente significativas entres os sexos.

Silva, Balaban e Motta, ${ }^{17}$ também realizaram estudo em Recife, onde avaliaram 230 escolares de 10 a 19 anos matriculados em uma escola da rede pública de Pernambuco, e encontraram resultados semelhantes aos nossos no que se refere à maior prevalência de sobrepeso no sexo masculino e maior prevalência de obesidade no sexo feminino.

Em São Paulo, Priore ${ }^{18}$ avaliou 320 adolescentes de 7 a 17 anos de escolas públicas da capital, onde foi encontrada maior prevalência de sobrepeso no sexo masculino, em concordância com o presente estudo.

Em Camboriú/SC, Beller ${ }^{19}$ realizou estudo com 105 adolescentes com idade entre 17 e 18 anos, sendo que a prevalência de sobrepeso foi maior para o sexo masculino em relação ao feminino. Em Natal, RN, Lyra $^{20}$ realizou estudo com 323 alunos, com idade entre 16 e 20 anos, matriculados em escolas públicas; a prevalência de sobrepeso foi maior para o sexo masculino do que para o sexo feminino. Ressalta-se que, embora as amostras avaliadas nos 
estudos de Beller ${ }^{19}$ e Lyra $^{20}$ e do presente estudo serem muito distintas em relação à faixa etária selecionada para o estudo, os resultados foram semelhantes.

Em estudo de Lamounier et al. ${ }^{21}$ na cidade de Belo Horizonte/MG, com 114 pacientes do Ambulatório de Adolescência do Hospital das Clínicas da UFMG, com média de idade de 15,6 anos, a prevalência de sobrepeso foi maior no sexo masculino em relação ao feminino, assim como no presente estudo.

Levando em consideração as semelhanças ou discrepâncias encontradas ao comparar o presente estudo com os artigos supracitados, realizados em todo o Brasil, deve-se ressaltar que o critério de classificação para o IMC não foi o mesmo, sendo que no presente estudo foi adotada a tabela proposta por Conde e Monteiro, ${ }^{9}$ enquanto que nos artigos revisados, utilizaram curvas de percentil; além de outros fatores e variáveis que não foram controladas e que podem ter vindo a interferir nos resultados, como: alimentação, prática de atividades físicas, clima, faixa etária da amostra, estilo de vida característico de cada região, entre outros.

Em Campo Grande/MS, Nunez et al. ${ }^{22}$ avaliaram 316 escolares entre 6 e 10 anos de idade, matriculados em um escola da rede pública estadual. Os resultados apontaram maior prevalência de sobrepeso e obesidade no sexo feminino, embora as diferenças não tenham sido estatisticamente diferentes. Ressalta-se que o critério de classificação do IMC adotado naquele estudo foi o mesmo utilizado por nós no presente estudo, ou seja, Conde e Monteiro. ${ }^{9}$ Os resultados apresentaram-se discrepantes apenas no sobrepeso, o que pode ser atribuído a inúmeros fatores, entre eles os regionais, por exemplo, ou até mesmo na faixa etária das amostras dos estudos, que não se assemelharam.

Em Florianópolis, um estudo foi realizado por Farias Júnior e Lopes, ${ }^{23}$ com amostra de 1.832 escolares do Ensino Médio, com idade entre 15 e 18 anos, com objetivo de descrever a prevalência de sobrepeso em adolescentes, a qual foi verificada com maior significância no sexo masculino, assim como no presente estudo, apesar de não ser utilizado o mesmo critério de classificação para o IMC e ao fato de as faixas etárias das amostras serem distintas. 
No tocante à prevalência de sobrepeso e obesidade entre os sexos, Guedes et al. ${ }^{24}$ avaliaram 4.319 alunos de 16 escolas da região de Apucarana/PR, com idade entre 7 e 18 anos e concluíram que a maior prevalência de obesidade se deu no sexo feminino em relação ao masculino, embora as diferenças não tenham sido estatisticamente significativas. Não foi encontrada semelhança quanto ao sobrepeso entre os resultados, pois no presente estudo a mesma foi verificada com maior significância no sexo masculino, enquanto que no estudo de Guedes et al., ${ }^{24}$ foi no sexo feminino. Ressalta-se aqui, que para ambas as amostras, não foram adotados os mesmos critérios de classificação do IMC, o que pode ter interferido nos resultados finais.

Ainda no Brasil, mais especificamente na região Oeste Catarinense, um trabalho realizado por Livinalli e Ribeiro, ${ }^{25}$ no município de Maravilha/SC, com 437 crianças escolares de 5 a 10 anos, encontrou maior prevalência tanto de sobrepeso, quanto de obesidade $(10,9 \%$ e $9,49 \%$, respectivamente) no sexo masculino, em comparação com o sexo feminino $(7,97 \%$ e $8,85 \%$, respectivamente), diferindo-se apenas quanto ao percentual de obesidade, o qual foi um pouco maior no sexo feminino no nosso estudo. Tal semelhança nos resultados pode ser atribuída a fatores como cultura e costumes parecidos, por serem amostras da mesma região.

Ao compararmos nosso estudo com pesquisas internacionais, encontramos uma publicação de Soveral et al., ${ }^{26}$ o qual avaliou 1.405 alunos de ambos os sexos, matriculados em 4 escolas públicas de Lisboa, em Portugal, com faixa etária entre 9 e 19 anos. O critério de classificação para o IMC adotado por Soveral et al. ${ }^{26}$ foi o mesmo adotado no presente estudo.

Os resultados encontrados, somando valores correspondentes de sobrepeso e obesidade, apontam uma maior prevalência de peso superior ao normal na população feminina da amostra. Analisando estes resultados, percebe-se que houve diferença em relação ao nosso estudo, o qual demonstrou maior prevalência de excesso de peso no sexo masculino e sem diferenças estatisticamente significativas entre os sexos quanto à prevalência de obesidade. Inúmeros fatores podem vir a explicar, em partes, a discrepância dos resultados, afinal, se trata de amostras de alunos com faixas etárias que não se igualam, além de pertencerem a 
locais totalmente distintos, com costumes diferentes e que podem vir a influenciar de forma significativa nos resultados.

De acordo com os estudos supracitados, os que apontaram similaridade com o presente estudo foram os de Balaban e Silva, ${ }^{16}$ Silva, Balban e Motta, ${ }^{17}$ Nunez et al., ${ }^{22}$ Guedes et al., ${ }^{24}$ Soveral et al., ${ }^{26}$ os quais apresentaram resultados elevados em relação a prevalência de sobrepeso e baixos no que se refere a prevalência de obesidade.

Apesar do fato de em nosso estudo algumas variáveis não terem sido controladas, tais como o nível de atividade física, hábitos alimentares, e outras, encontramos resultados significantes em nosso estudo e que vão ao encontro do que as evidências atuais apontam, ou seja, que o fenômeno do sobrepeso e da obesidade, antes restrita aos países desenvolvidos, tem ocorrido em nosso meio em uma velocidade alarmante. A globalização, as facilidades da sociedade moderna, a progressiva substituição das comidas tradicionais e dos hábitos de vida saudáveis, juntamente com o acesso à tecnologia, propiciam que os adolescentes sejam mantidos em suas casas, o que tem levado cada vez mais ao sedentarismo, elevando a prevalência de adolescentes com sobrepeso e obesidade.

\section{CONCLUSÃO}

O presente estudo conclui que, em ambos os sexos, a prevalência de sobrepeso e obesidade nos escolares com idade de 7 a 17 anos, residentes nos municípios que fazem parte da SDR de São Miguel do Oeste/SC, é considerada alta, pois atinge uma fração de $24,8 \%$ da população feminina e $26,4 \%$ da masculina.

Ao comparar os sexos, verificou-se que a prevalência de sobrepeso foi maior nos escolares do sexo masculino do que no sexo feminino, principalmente nos municípios de Belmonte/SC, Descanso/SC e São Miguel do Oeste/SC enquanto que, não foram observadas diferenças significativas entre os sexos no que se refere à prevalência de obesidade.

Enfim, estes resultados justificam a necessidade da elaboração e implementação de um programa de intervenção nas escolas dos municípios da SDR de São Miguel do Oeste, principalmente, naquelas em que os índices foram mais alarmantes quanto à prevalência de 
sobrepeso e obesidade. Sugere-se que novos estudos sejam realizados e que sejam controlados os fatores associados ao sobrepeso e obesidade.

\section{REFERÊNCIAS}

${ }^{1}$ BOUCHARD, C. Atividade física e obesidade. Barueri: Manole, 2003.

${ }^{2}$ MCARDLE, W. D.; KATCH, F. I.; KATCH, V. L. Fisiologia do exercício: energia, nutrição e desempenho humano. 5. ed. Rio de Janeiro: Guanabara Koogan, 2003.

${ }^{3}$ VIUNISKI, N. Obesidade infantil: guia prático. 2. ed. Rio de Janeiro: EPUP, 1999.

${ }^{4}$ ANDERSON, P. M.; BUTCHER, K. F. Reading, writing and refreshments: are school finances contributing to children's obesity?. Journal of Human Resources, Madison, v. 41, n. 3, p. 467-494, $2006 . \quad$ Disponível em: <http://www.dartmouth.edu/ pmaweb/RaisinetsOct2005.pdf $>$. Acesso em: 30 jun. 2011.

${ }^{5}$ LAMOUNIER, J. A.; ABRANTES, M. M. Prevalência de obesidade e sobrepeso na adolescência no Brasil. Revista Médica de Minas Gerais, Belo Horizonte, v.13, n. 4, p. 275-284, 2003. Disponível em: <http://www.medicina.ufmg.br/observaped/images/docs/prevalencia_obesidade_sobrepeso _criancas_brasil_rmmg_artigo.pdf>. Acesso em: 19 jun. 2011.

${ }^{6}$ SILVA, Y. M. P.; COSTA, R. G.; RIBEIRO, R. L. Obesidade infantil: uma revisão bibliográfica. Saúde e Ambiente em Revista, Rio de Janeiro, v. 3, n. 1, p. 01-15, 2008. Disponível em: <http://publicacoes.unigranrio.edu.br/index.php/sare/article/view/215>. Acesso em: 15 jun. 2011.

${ }^{7}$ SOARES, L. D.; PETROSKI E. L. Prevalência, fatores etiológicos e tratamento da obesidade infantil. Revista Brasileira de Cineantropometria e Desempenho Humano, Florianópolis, v. 5, n.1, p. 63-74, 2003. Disponível em: <http://www.rbcdh.ufsc.br/DownloadArtigo.do\%3Bjsessionid=E8C866A75126F2F02E07 7194E3AA572D? artigo=104>. Acesso em: 29 jun. 2011. 
${ }^{8}$ GAYA, A.; SILVA, G. Projeto Esporte Brasil: manual de aplicação de medidas e testes, normas e critérios de avaliação. 2007. p. 1-28. Disponível em: <http://www.proesp.ufrgs.br/institucional/MANUAL\%20PROESP-BR\%20\%20JULHO\%20DE\%202007.pdf>. Acesso em: 18 mar. 2011.

${ }^{9}$ CONDE, W. L.; MONTEIRO, C. A. Valores críticos do índice de massa corporal para classificação do estado nutricional de crianças e adolescentes brasileiros. Jornal de Pediatria, Rio de Janeiro, v. 82, n. 4, p. 266-272, 2006. Disponível em: $<$ http://www.scielo.br/scielo.php?script=sci_arttext\&pid=S002175572006000500007\&lng=en\&nrm=iso >. Acesso em: 22 jun. 2011.

${ }^{10} \mathrm{SOAR}, \mathrm{C}$. et al. Prevalência de sobrepeso e obesidade em escolares de uma escola pública de Florianópolis, Santa Catarina. Revista Brasileira de Saúde Materno Infantil, Recife, v. 4, n. 4, p. 391-397, 2004. Disponível em: 〈http://www.scielo.br/pdf/rbsmi/v4n4/a08v04n4.pdf >. Acesso em: 17 jun. 2011.

${ }^{11}$ COLE, T. J. et al. Establishing a standard definition for child overweight and obesity worldwide: international survey. British Medical Journal, v. 320, n. 1744, p.1240-1243, 2000.

${ }^{12}$ SOUZA LEÃO, L. S. C. et al. Prevalência de obesidade em escolares de Salvador, Bahia. Arquivo Brasileiro de Endocrinologia Metabólica, v. 47, n. 02, p.151-157, 2003. Disponível em: 〈http://www.scielo.br/pdf/abem/v47n2/a07v47n2.pdf > Acesso em: 17 jun. 2011.

${ }^{13}$ HIMES, J. H.; DIETZ, W. H. Guidelines for overweight in adolescent preventive services recommendations from an Expert Committee. American Journal of Clinical Nutrition, New York, v. 59, n. 2, p. 307-316, 1994.

${ }^{14}$ NEUTZLING, M. B. et al. Overweight and obesity in Brazilian adolescents. Journal of International Obesity Metabolism Disorders, v. 24, n. 7, p. 869-874, 2000. Disponível 
em: 〈http://www.nature.com/ijo/journal/v24/n7/abs/0801245a.html>. Acesso em: 14 jun. 2011.

${ }^{15}$ FONSECA, V. M.; SICHIERI, R.; VEIGA, G. V.. Fatores associados à obesidade em adolescentes. Revista Saúde Pública, São Paulo, v. 32, n. 6, p.1-10, 1998. Disponível em: <http://www.scielo.br/scielo.php?script=sci_arttext\&pid=S0034-89101998000600007>. Acesso em: 18 jun. 2011.

${ }^{16}$ BALABAN, G.; SILVA, G. A. P. Prevalência de sobrepeso e obesidade em crianças e adolescentes de uma escola da rede privada de Recife. Jornal de Pediatria, Rio de Janeiro, v. 77, n. 2, p. 96-100, 2001. Disponível em: <http://www.scielo.br/pdf/jped/v77n2/v77n2a08.pdf>. Acesso em: 18 jun. 2011.

${ }^{17}$ SILVA, G. A. P. ; BALABAN, G.; MOTTA, M. E. F. A. Prevalência de sobrepeso e obesidade em adolescentes de uma escola da rede pública do Recife. Revista Brasileira de Saúde Materno Infantil, Recife, v. 2, n. 1, p. 37-42, 2002. Disponível em: $<$ http://www.scielo.br/scielo.php?pid=S151938292002000100006\&script=sci_pdf\&tlng=pt $>$. Acesso em: 29 jun. 2011.

${ }^{18}$ PRIORE, S. E. Composição corporal e hábitos alimentares de adolescentes: uma contribuição à interpretação dos indicadores do estado nutricional. 1998. 211f. Tese (Doutorado em Medicina) - Universidade Federal de São Paulo, São Paulo, 1998.

${ }^{19}$ BELLER, F. S. Alimentação e estilo de vida de adolescentes em balneário Camboriú, Santa Catarina, Brasil. In: CONGRESSO LATINOAMERICANO DE NUTRICIÓN, 12., Buenos Aires, 2000. Libro de resúmenes de trabajos libres... Buenos Aires, 2000, 12-16 nov. 2000. p. 224.

${ }^{20}$ LYRA, C. O. O estado nutricional em escolares adolescentes de Natal/RN: um inquérito de prevalência. 2000. 73f. Dissertação (Mestrado em Saúde Coletiva) - Instituto de Medicina Social, Universidade do Estado do Rio de Janeiro, Rio de Janeiro, 2000. 
${ }^{21}$ LAMOUNIER, J. A. et al. Prevalência de sobrepeso em adolescentes no ambulatório de adolescência do Hospital das Clínicas da UFMG. In: SIMPÓSIO OBESIDADE E ANEMIA CARENCIAL NA ADOLESCÊNCIA, Salvador, 2000. Anais... Salvador, 8-9 de junho de 2000. p. 211-212.

${ }^{22}$ NUNEZ, P. R. M. et al. Análise do índice de massa corporal de escolares de 6 a 10 anos residentes em Campo Grande - MS e a diferença entre sexo. Conexões: revista da Faculdade de Educação Física da UNICAMP, Campinas, v. 6, n. 3, p. 01-10, 2008. Disponível

em: $<$ http://polaris.bc.unicamp.br/seer/fef/include/getdoc.php?id=1169\&article=419\&mode=pd f>. Acesso em: 29 jun. 2011.

${ }^{23}$ FARIAS JÚNIOR, J. C.; LOPES, A. S. Prevalência de sobrepeso em adolescentes. Revista Brasileira Ciência e Movimento, Brasília, v. 11, n. 3, p. 77-84, 2003. Disponível em: <http://www.ucb.br/mestradoef/RBCM/11/11\%20-\%203/c_11_3_12.pdf >. Acesso em: 29 jun. 2011.

${ }^{24}$ GUEDES, D. P. et al. Prevalência de sobrepeso e obesidade em crianças e adolescentes: estimativas relacionadas ao sexo, à idade e à classe socioeconômica. Revista Brasileira de Educação Física e Esporte, São Paulo, v. 20, n. 3, p.151-163, 2006. Disponível em: < http://www.usp.br/eef/rbefe/v20n32005/1_Dartagnan.pdf>. Acesso em: 30 jun. 2011.

${ }^{25}$ LIVINALLI, E. F; RIBEIRO, A. J. P. Influência do nível socioeconômico e dos hábitos alimentares no desenvolvimento de sobrepeso e obesidade em crianças de 5 a 10 anos estudantes do município de Maravilha/SC. 2006. Trabalho de Conclusão de Curso (Licenciatura) - Universidade do Oeste de Santa Catarina, São Miguel do Oeste, Santa Catarina, 2006.

${ }^{26}$ SOVERAL, G. et al. Estilo de vida e risco de obesidade em adolescentes na região de Lisboa. Revista Lusófona de Ciências e Tecnologia da Saúde, v. 2, n. 2, p.109-115, 2005 . Disponível em:

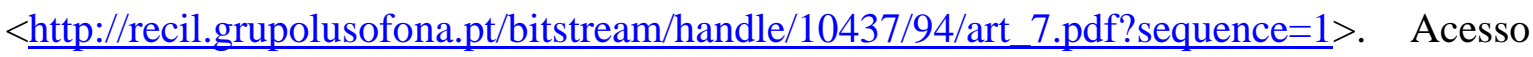
em: 19 jun. 2011.

Conexões: revista da Faculdade de Educação Física da UNICAMP, Campinas, v. 11, n. 1, p. 57-73, jan./mar. 2013.72 ISSN: 1983-9030 
Recebido em: 07 dez. 2012

Aceito em: 01 mar. 2013

Contato: Andréa Jaqueline Prates Ribeiro andrea.ribeiro@unoesc.edu.br 